\title{
APPLICATION OF GREEN EXTRACTION METHODS TO RESVERATROL EXTRACTION FROM PEANUT (ARACHIS HYPOGAEA L.) SKIN
}

\author{
REZI RIADI SYAHDI ${ }^{1}$, RATNA NADYANA ${ }^{1}$, RIZQI HERU PUTRI ${ }^{1}$, ROSEDIANA SANTI ${ }^{2}$, ABDUL MUN'IM ${ }^{1 *}$
}

${ }^{1}$ Department of Pharmacognosy Phytochemistry, Faculty of Pharmacy, Universitas Indonesia, Depok, Indonesia. ${ }^{2}$ Department of Biomedical Computation, Faculty of Pharmacy, Universitas Indonesia, Depok, Indonesia. Email: munim@farmasi.ui.ac.id

Received: 02 October 2019, Revised and Accepted: 24 December 2019

\section{ABSTRACT}

Objective: This study compared the maceration extraction method with non-conventional extraction methods such as ultrasound-assisted extraction (UAE) and microwave-assisted extraction (MAE).

Methods: To obtain resveratrol, various conditions were optimized: Solvent types (organic solvent [i.e. 70\% ethanol] and green solvents [i.e. natural deep eutectic solvent (NADES) and ionic liquid (IL)]) and extraction methods. The resveratrol content in the extracts was analyzed by high-performance liquid chromatography.

Results: It was determined that resveratrol extracted by UAE with NADES was composed of choline chloride-oxalic acid when the following conditions were used: The solid/liquid ratio of $1: 20(\mathrm{~g} / \mathrm{mL})$ and the extraction time of $15 \mathrm{~min}$. These conditions produced higher resveratrol content $(0.049 \mathrm{mg} / \mathrm{g}$ dry weight) than that using MAE with $70 \%$ ethanol $(0.011 \mathrm{mg} / \mathrm{g}$ dry weight). However, the maceration method yielded the highest amount of resveratrol $(0.221 \mathrm{mg} / \mathrm{g}$ dry weight $)$, and MAE with IL produced the smallest amount of resveratrol $\left(0.157 \times 10^{-3} \mathrm{mg} / \mathrm{g} \mathrm{dry} \mathrm{weight}\right)$.

Conclusion: Peanut skin extracted using the maceration method produced the highest amount of resveratrol compared to that using other methods.

Keywords: Arachis hypogaea, Extraction, Ionic liquid, Natural deep eutectic solvent, Resveratrol.

(C) 2020 The Authors. Published by Innovare Academic Sciences Pvt Ltd. This is an open access article under the CC BY license (http://creativecommons. org/licenses/by/4. 0/) DOI: http://dx.doi.org/10.22159/ijap.2020.v12s1.FF003

\section{INTRODUCTION}

Resveratrol is a polyphenolic compound, which was initially classified as stilbene phytoalexins. Resveratrol possesses many biological activities. It compound acts by inhibiting oxidative reactions and is used to treat hyperlipidemia due to its HMG-CoA reductase inhibitory activity [1-3]. The compound is present in many plant species (e.g., grapes and mulberries) and peanut skin [4]. The latter is the by-product of peanut seed production. Peanut skin has been reported as the source of resveratrol, which can be obtained using a suitable solvent and extraction method. The extraction of resveratrol from peanut skin has been achieved by the maceration method using ethanol as a solvent [5]. However, conventional extraction methods have disadvantages such as long extraction time and large solvent consumption [6]. Recently, resveratrol from peanut skin has been extracted by modern extraction methods such as microwave-assisted extraction (MAE) with ethanol [7].

Modern extraction methods and green solvents have been developed to extract bioactive compounds effectively and efficiently. Green solvents such as ionic liquids (ILs) are used in various extraction processes because of their unique properties [8]. Recently, it has been reported that IL [bmim]Br extracts trans-resveratrol from Rhizoma Polygoni Cuspidati and Melinjo seeds more effectively than organic solvents $[9,10]$. The IL-MAE method was chosen in this study because IL possesses good dissolution property and absorbs more microwave radiation than organic solvents [8]. In this research, the IL-MAE method used urea as a pre-treatment material. Peanut skin contains cellulose (40.5\%), lignin (26.4\%), and hemicellulose (14.7\%) [11]. Urea pretreatment has been reported to remove lignin and hemicellulose, which improves solvent accessibility to dissolve active compounds [12].

Another green solvent, such as natural deep eutectic solvents (NADES), is effective at extracting polar and non-polar compounds [13]. It has been reported that choline chloride $(\mathrm{ChCl})$ and its hydrogen bond donors (HBDs) (e.g., oxalic acid, malic acid, and glycerol) are effective at extracting anthocyanin from grape skin [14]. Furthermore, ureaglycerol as NADES combined with the ultrasound-assisted extraction (UAE) method has been reported to increase the amount of polyphenol compounds extracted from green tea leaves [15]. In this research, oxalic acid, malic acid, and glycerol served as NADES HBD, and $\mathrm{ChCl}$, and urea served as hydrogen bond acceptor (HBA).

This research aims to compare the effectiveness of resveratrol extraction with two extraction methods (UAE and MAE) with green solvents. The resveratrol extract was quantitatively analyzed by highperformance liquid chromatography (HPLC), and the mechanism of extraction was determined by scanning electron microscopy (SEM). On the basis of this research, an extraction method can be suggested to better extract resveratrol using the UAE and MAE methods with IL and NADES as solvents.

\section{MATERIALS AND METHODS}

\section{Chemicals and materials}

Choline chloride (>99\%) was purchased from Xi'an Rongsheng Biotechnology (China), and 1-butyl-3-methylimidazolium bromide ([bmim]Br) was purchased from Shanghai Cheng Jie Chemical (China). Oxalic acid, glycerol, sorbitol, propylene glycol, and demineralized water were purchased from Dow Chemical Pacific (Singapore). Sodium carbonate (Sigma-Aldrich, USA), urea, malic acid, methanol, ethanol, and ethyl acetate were used for the analysis (Merck, Germany). Acetonitrile, methanol, and acetic acid (Merck, Germany) were of HPLC grade, and standard resveratrol was purchased from Wako Pure Chemical Industries (Japan).

\section{Instrumentation}

Modena microwave with modification, maceration vessel, ultrasonic bath (Krisbow, China), rotary vacuum evaporator (Buchi, Germany), hot 
plate (IKA C-MAG HS 4, Germany), HPLC (Shimadzu LC-20AT, Japan), GL Sciences C-18 column (Japan), microsyringe (Hamilton, Germany), and Design-Expert 10.0.3 software were used for the IL-MAE data collection, processing, and analysis.

\section{Preparation of raw materials}

Peanut seeds were obtained from Groundnut Farming in Cilegon, Banten, Indonesia, and identified at Lembaga Ilmu Pengetahuan Indonesia, Bogor, West Java, Indonesia. Peanut skin was separated and cleaned from its other parts. The hard outer skin was peeled and dried at room temperature. After drying, the dried material was ground using an electric grinder, and the resulting powder was sieved through a 20mesh sieve. The dried powder was stored in a sealed container to avoid exposure to air and direct sunlight.

\section{Preparation of NADES}

NADES used in this study are mixtures of $\mathrm{ChCl}$ or urea and HBD (oxalic acid, malic acid, and glycerol). The chemical composition and mixing ratios of individual chemicals of synthesized NADESs are listed in Table 1. These NADES were prepared by heating the mixtures consisting of solid-liquid and solid-solid compounds at $50^{\circ} \mathrm{C}$ and $80^{\circ} \mathrm{C}$ with constant stirring, respectively. Stirring was continued for 30-90 min until a clear homogeneous liquid solution formed [16].

\section{Extraction process}

Conventional maceration method

The dried powders were extracted in $70 \%$ ethanol $(50 \mathrm{~mL} / 2.5 \mathrm{~g})$ for $24 \mathrm{~h}$ and shaken for $1 \mathrm{~h}$ in a dark container at room temperature. The extracts were filtered and vacuum-evaporated to obtain a crude extract. Furthermore, the residue was extracted using the same procedure two more times. The yield of the extracts was calculated according to the following formula:

$$
\text { Yield }(\%)=\frac{\text { Amount extract obtained }(\mathrm{g})}{\text { Dry weight }(\mathrm{g})} \times 100 \%
$$

\section{MAE method}

The dried powder (1.5 g) was placed in a $100-\mathrm{mL}$ flat flask, and $22.5 \mathrm{~mL}$ of $70 \%$ ethanol was added to it. The dried peanut skin powder was extracted using MAE with a 30-s irradiation time. The extraction variable evaluated was microwave power (e.g. 270, 450, and $630 \mathrm{~W}$ ). The crude extract was obtained after evaporating solvent at $50^{\circ} \mathrm{C}$ using a rotary evaporator

\section{IL-based MAE method}

The dried powder $(1 \mathrm{~g})$ was weighed and put into a $100-\mathrm{mL}$ flat flask and then dissolved in $20 \mathrm{~mL}$ of $2.5 \mathrm{M} \mathrm{IL}$ [bmim] Br. The samples were extracted using MAE with a 270-W microwave power.

\section{IL-based MAE (IL-MAE) method with urea pre-treatment}

The dried peanut skin powder (1 g) was soaked in $20 \mathrm{~mL}$ of urea solution with five various concentrations (e.g., 5, 10, 15, 20, and $25 \% \mathrm{w} / \mathrm{v}$ ) and pre-cooled at $-20^{\circ} \mathrm{C}$ for $20 \mathrm{~min}$. These solutions were pre-treated for $12 \mathrm{~h}$ at $23^{\circ} \mathrm{C}$. Then, $20 \mathrm{~mL}$ of $2.5 \mathrm{M} \mathrm{IL}$ [bmim] Br was added to each solution, and these solutions were extracted using MAE with a 270-W microwave power. The urea concentration, which produced the best resveratrol content, was chosen and optimized using response surface methodology (RSM).

\section{NADES-based UAE (NADES-UAE) method}

The extraction of resveratrol using NADES was carried out by mixing $1 \mathrm{~g}$ of a peanut skin powder with $20 \mathrm{~mL}$ of NADES in sealed vials at room temperature. Then, the mixture was centrifuged for $1 \mathrm{~h}$ at $3000 \mathrm{rpm}$ and filtered through a filter paper to obtain the supernatant.

\section{HPLC analysis}

HPLC analysis followed the Souto et al. method with some modifications. The chromatographic method was performed using the Shimadzu HPLC system equipped with an LC-20AT pump, UV-vis detector, microsyringe with a $25-\mu \mathrm{L}$ loop, and a C-18 column $(150 \mathrm{~mm} \times 4.6 \mathrm{~mm}$ long, with a $5-\mu \mathrm{m}$ particle diameter). Reversed-phase HPLC was performed with an isocratic elution ( $1 \mathrm{~mL} / \mathrm{min}$ flow rate) at room temperature. The eluent consisted of water and acetonitrile $(75: 25, \mathrm{v} / \mathrm{v})$, and the $\mathrm{pH}$ of the solution was adjusted to 3.0 by adding a $0.5 \%$ acetic acid solution. The mobile phase was filtered with a vacuum filter and sonicated before use. The UV-vis detector wavelength was set at $306 \mathrm{~nm}$. Before injection, the sample was first diluted with methanol and filtered using a $0.45-\mu \mathrm{m}$ microporous membrane (Shimadzu, Japan). The injection amount was $20 \mu \mathrm{L}$, and the total elution time was $15 \mathrm{~min}$. Each sample was injected in triplicate. Resveratrol in the peanut skin extracts was initially identified by comparing the retention times and UV-vis spectra of the samples to a resveratrol standard, which was analyzed under the same chromatographic conditions. Resveratrol content was expressed as mg per gram of dry peanut skin [17].

Scanning electron microscopy analysis of the peanut skin powder The peanut skin powder was imaged directly before extraction. After extraction, the peanut skin powder was dried to remove the solvent before imaging.

\section{RESULTS}

\section{Resveratrol content}

The extract produced by the maceration method contained the highest amount of resveratrol $(0.221 \mathrm{mg} / \mathrm{g}$ dry weight $)$, and the extract obtained by MAE using IL contained the lowest amount of resveratrol $\left(0.157 \times 10^{-3} \mathrm{mg} / \mathrm{g}\right.$ dry weight $)$.

\section{Extraction mechanism analysis}

The particle surface of the treated samples exhibited different morphology compared to untreated samples. All of the extraction methods destroy the physical structure of the peanut skin powder with different mechanism.

\section{DISCUSSION}

\section{Preparation of NADES}

NADES were synthesized by mixing many pairs of HBDs and HBAs at various molar ratios using the heating and mixing method. Six different types of NADES, including three choline-based and three urea-based NADESs, were successfully synthesized as clear and stable liquids. These NADES produced the same results as in the previous studies by Bubalo, Ćurko, Tomašević, Kovačević Ganić and Redovniković, 2016 and Jeong et al., 2017.

\section{HPLC analysis}

Calibration of standard resveratrol

The salt compound $(\mathrm{ChCl})$ and HBD were weighed st a determined ratio (Table 2) and mixed. Then, the mixture was stirred and heated for $30-90 \mathrm{~min}$ in a hotplate at $50^{\circ} \mathrm{C}$ for the solid-liquid compound combinations and at $80^{\circ} \mathrm{C}$ for the solid-solid mixture until a clear solution was formed [12].

\section{Resveratrol content}

The resveratrol assay was determined by HPLC. Fig. 1 shows the chromatogram of resveratrol and ethanol extract produced by the

Table 1: NADES combinations and mole ratios used in this study

\begin{tabular}{lllll}
\hline No & Code & HBA & HBD & HBA: HBD mole ratio \\
\hline 1 & NADES-1 & ChCl & Oxalic acid & $1: 1$ \\
2 & NADES-2 & ChCl & Malic acid & $1: 1$ \\
3 & NADES-3 & ChCl & Glycerol & $1: 2$ \\
4 & NADES-4 & Urea & Glycerol & $1: 2$ \\
5 & NADES-5 & Urea & Sorbitol & $1: 2$ \\
6 & NADES-6 & Urea & Propylene glycol & $1: 2$ \\
\hline
\end{tabular}

HBA: Hydrogen bond acceptor, NADES: Natural deep eutectic solvents, HBD: Hydrogen bond donors, $\mathrm{ChCl}$ : Choline chloride 


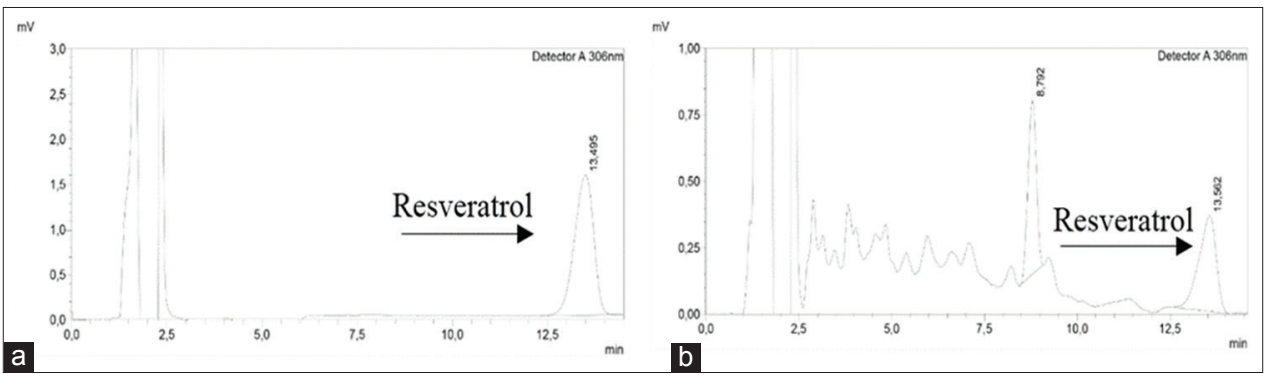

Fig. 1: (a) High-performance liquid chromatography of resveratrol $(0.2 \mathrm{ppm})$ and the (b) ethanol extract produced by the maceration method

Table 2: Overview of extraction methods and comparison of the resveratrol content in the peanut skin extract

\begin{tabular}{lll}
\hline Extraction method & Solvent & $\begin{array}{l}\text { Concentration } \\
\text { (mg/g dry weight) }\end{array}$ \\
\hline Maceration & $70 \%$ ethanol & 0.221 \\
MAE & $70 \%$ ethanol & 0.011 \\
MAE & [bmim] Br & $0.157 \times 10^{-3}$ \\
MAE & [bmim] Br with & $0.290 \times 10^{-3}$ \\
& urea pre-treatment & \\
UAE & NADES-1* & 0.049 \\
UAE & NADES-2* & 0.021 \\
UAE & NADES-3* & 0.023 \\
UAE & NADES-4* & 0.023 \\
UAE & NADES-5* & 0.020 \\
UAE & NADES-6* & 0.025 \\
\hline
\end{tabular}

*Explanation in Table 1. MAE: Microwave-assisted extraction,

UAE: Ultrasonic-assisted extraction, NADES: Natural deep eutectic solvent

maceration method. The resveratrol content of each extract is presented in Table 2. The extract produced by the maceration method contained the highest amount of resveratrol, and the extract obtained by MAE using IL contained the lowest amount of resveratrol.

Table 2 shows that among all extraction methods, maceration with $70 \%$ ethanol yielded the highest resveratrol content $(0.221 \mathrm{mg} / \mathrm{g}$ dry weight). This result was obtained owing to the effectiveness of ethanol as a solvent and the length of extraction time. Ethanol is less viscous than NADES and IL [18]. Thus, the lower viscosity of the solvent increases the diffusion coefficient, which increases the extraction rate. According to the Stokes-Einstein equation, viscosity $(\eta)$ affects the diffusion coefficient $(D)$.

$$
D=\frac{k T}{6 \Pi \eta r}
$$

From the abovementioned equation, where $\eta$ is inversely related to $D$, it can be assumed that ethanol, which is less viscous than NADES and IL, has a higher diffusivity. The high diffusion coefficient value of ethanol made the particles of dried powder move and distribute easily in ethanol than in NADES and IL [18].

However, during the extraction using MAE with 70\% ethanol, a lower resveratrol content was obtained $(0.011 \mathrm{mg} / \mathrm{g}$ dry weight $)$ than that using maceration with $70 \%$ ethanol. This result was possibly obtained owing to the selection of less optimum time. Longer extraction time allows more contact between the solvent and sample, which increases solvent absorption, helps soften plant tissues, ruptures plants cell walls, and increases the solubility of the target compound in the solvent [19]. However, excessive exposure to microwave radiation decreases the extraction results due to the deterioration of the target compound [20].

In this study, urea pre-treatment aimed to improve solvent accessibility through the cell wall so that a higher amount of the active compound could be extracted. Based on the results using five urea concentrations,
$15 \% \mathrm{w} / \mathrm{v}$ was selected. After the pre-treatment for $12 \mathrm{~h}$, the sample powder swelled. The cell wall was stretched for further treatment with the IL-MAE method, as suggested by a previous similar study [21]. However, both extraction methods (i.e., with and without urea pretreatment) yielded the lowest amounts of resveratrol (i.e., $2.9 \times 10^{-4} \mathrm{mg} / \mathrm{g}$ dry weight and $1,57 \times 10^{-4} \mathrm{mg} / \mathrm{g}$ dry weight, respectively). The profile chromatograms of both samples (Fig. 2) show that higher amounts of non-target compounds, which are more polar than the target compound, are extracted. Moreover, RSM results predicted that there were no optimum conditions for extracting resveratrol using IL-MAE with three parameters, including the IL concentration $(0.5,1.5$, and 2.5 $\mathrm{M})$, extraction time $(10,13$, and $16 \mathrm{~min})$, and liquid/solid ratio $(10,15$, and $20 \mathrm{~mL} / \mathrm{g}$ ).

Moreover, the data obtained for the extraction using the NADES-UAE method with $\mathrm{ChCl}$ as $\mathrm{HBA}$ clearly show that the mixtures of $\mathrm{ChCl}$ in combination with oxalic acid (NADES-1) are able to extract higher amounts of resveratrol $(0.049 \mathrm{mg} / \mathrm{g}$ dry weight $)$ compared to those obtained with the other types of NADES using the following conditions: The solid/liquid ratio of $1: 20(\mathrm{~g} / \mathrm{mL})$ and the extraction time of $15 \mathrm{~min}$. Meanwhile, for NADES with urea as HBA, the highest resveratrol content was obtained by NADES composed of urea and propylene glycol ( $0.025 \mathrm{mg} / \mathrm{g}$ dry weight) using the following conditions: The solid/ liquid ratio of $1: 20(\mathrm{~g} / \mathrm{mL})$ and the extraction time of $10 \mathrm{~min}$.

Factors that may affect the extraction results include the physicochemical properties of NADES, such as solubility, viscosity, surface tension, polarity, and hydrogen bond interactions between NADES and the target compound [22]. In terms of polarity, NADES-based organic acids are the most polar [16], which explains the highest resveratrol content obtained by NADES-1 compared with that obtained by another NADES types. However, NADES-based polyalcohol (NADES-6) with low polarity tends to yield lower amounts of resveratrol compared with that obtained with NADES that contains oxalic acid (NADES-1), as shown in Table 2.

In addition, the positioning of the hydroxyl group branches along the alkane chain [23] has implications for the surface tension of NADES. The target compound resveratrol is structurally similar to HBD; thus, it can be considered a type of HBD [23]

Therefore, HBDs with more branches bind stronger to HBA, which causes more steric hindrances that may inhibit the interaction of active compounds with HBAs [23]. Thus, polyalcohol and organic acids, which act as HBDs, will interact competitively by causing steric hindrance and inhibiting resveratrol from binding to the anions of urea or $\mathrm{ChCl}$, which act as HBAs.

Steric hindrance caused the formation of a rigid conformation, which resulted in high surface tension on NADES and made it difficult for the active compound to penetrate and form hydrogen bonds with HBA [18]. This explains why the extraction with NADES-1 produced the highest resveratrol content. Specifically, oxalic acid has the simplest (shortest chain) structure and contains hydroxyl groups that are not positioned near each other. This orientation results in a less dense conformation and low surface tension on NADES, which makes it easier to form bonds between the active compound and the anions in NADES. 


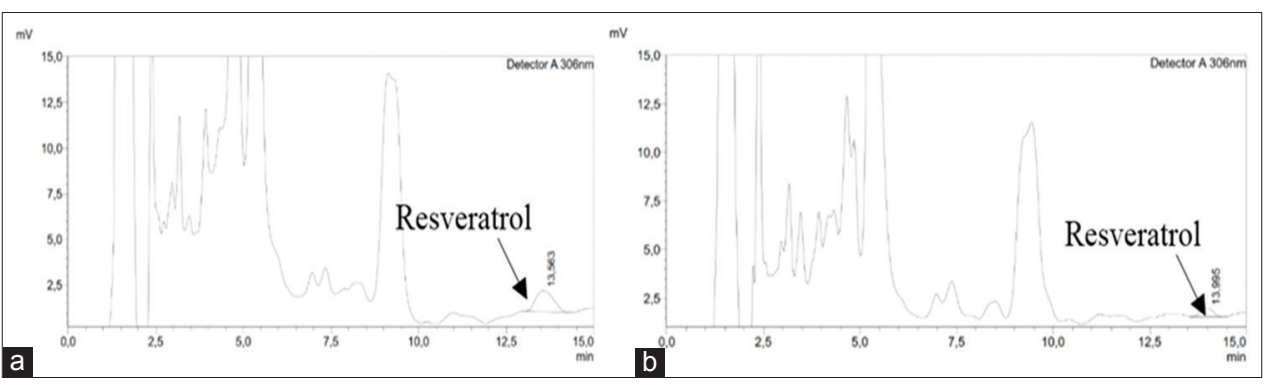

Fig. 2: (a) Chromatogram profiles of the extracts obtained using (a) ionic liquid-microwave-assisted extraction (IL-MAE) and (b) IL-MAE with urea pre-treatment
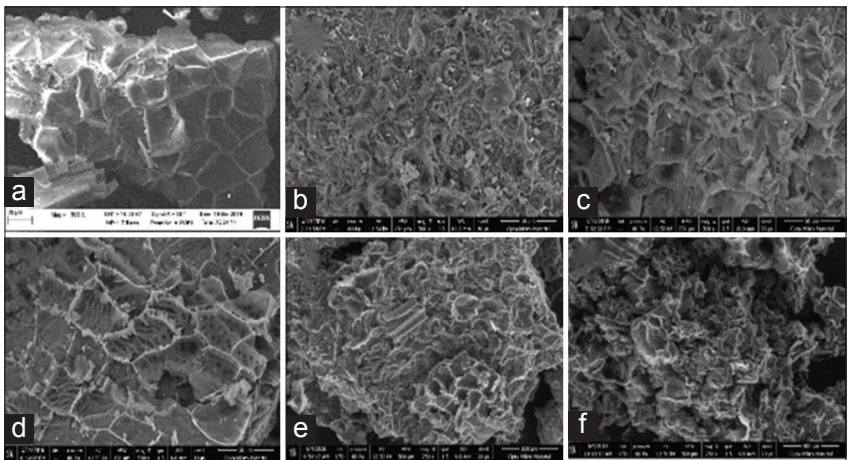

Fig. 3: Scanning electron microscopy image of the peanut skin powder (a) before treatment; (b) after maceration using $70 \%$ ethanol; (c) after microwave-assisted extraction (MAE) using 70\% ethanol; (d) after ultrasonic-assisted extraction using natural deep eutectic solvent choline chloride-oxalic acid; (e) after MAE using ionic liquid (IL) [bmim] Br; (f) after MAE using IL [bmim] Br with urea pre-treatment

Scanning electron microscopy analysis of the peanut skin powder To better understand the mechanism of different extraction methods, the peanut skin powder was examined using SEM before and after the extraction. Fig. 3 shows the particle surfaces before and after the maceration extraction, MAE using 70\% ethanol, MAE using IL with/ without urea pre-treatment, and UAE using NADES. Compared to the untreated sample (Fig. 3a), the particle surface of the treated samples exhibited different morphology. The maceration extraction method with $70 \%$ ethanol ruptured and destroyed the physical structure of the peanut skin powder (Fig. 3b). After prolonged extraction using organic solvents, such as ethanol, the peanut skin powder exhibits severely damaged morphology [15]. The purpose of the process is to soften and break the cell wall of the plant to release soluble phytochemicals [24]. The surface of the powder after the extraction using MAE with $70 \%$ ethanol was slightly ruptured (Fig. 3c). Microwave radiation has destructive effects on the matrix of the extracted sample due to the sudden increase in temperature and internal pressure inside the cells of the plant sample [25]. After the extraction using UAE with NADES, the particle surface was rugged and porous, and fibrous tissues were partially exposed (Fig. 3d). A possible mechanism for extraction enhancement could be that the implosion of cavitation bubbles on the leaves surface induces the erosion of plant structures, which are released in the extraction medium [26]. The sample surface after MAE using IL (Fig. 3e) was not considerably changed compared to that of the untreated sample. The internal thermal stress in the cell was not sufficiently strong to break down the cell wall; thus, the active compound was less extracted. However, the sample surface after using IL with urea pre-treatment (Fig. 3f) showed the enlargement of cellular pores. The loss of lignin and hemicellulose after urea pre-treatment did not protect cellulose from the heat and internal pressure produced by microwave radiation, which facilitated the leaching of the active compound [27]

\section{CONCLUSION}

On the basis of the obtained results, it was concluded that the extraction of peanut skin using the maceration method yielded the highest amount of resveratrol compared to that produced by other methods.

\section{ACKNOWLEDGMENTS}

This study was financially supported by the Directorate of Research and Community Engagement, Universitas Indonesia through HIBAH PITTA 2018.

\section{CONFLICTS OF INTEREST}

The authors have no conflicts of interest to declare.

\section{REFERENCES}

1. Cho IJ, Ahn JY, Kim S, Choi MS, Ha TY. Resveratrol attenuates the expression of HMG-CoA reductase mRNA in hamsters. Biochem Biophys Res Commun 2008;367:190-4

2. Hafidz KA, Puspitasari N, Azminah, Yanuar A, Artha Y, Mun'im A. HMG-CoA reductase inhibitory activity of Gnetum gnemon Seed extract and identification of potential inhibitors for lowering cholesterol level. J Young Pharm 2017;9:559-65.

3. Shimizu-Ibuka A, Udagawa H, Kobayashi-Hattori K, Mura K, Tokue C, Takita T, et al. Hypocholesterolemic effect of peanut skin and its fractions: A case record of rats fed on a high-cholesterol diet. Biosci Biotechnol Biochem 2009; 73:205-8

4. Lim TK. Edible Medicinal and Non-medicinal Plants: Fruits. Vol. 2. Netherlands: Springer; 2012. p. 513-540.

5. Nepote V, Grosso NR, Guzman CA. Optimization of extraction of phenolic antioxidants from peanut skins. J Sci Food Agric 2005;85:33-8.

6. Sarker S, Latif Z, Gray A. Natural Products Isolation. Totowa: Humana Press; 2005

7. Ballard TS, Mallikarjunan P, Zhou K, O'Keefe S. Microwave-assisted extraction of phenolic antioxidant compounds from peanut skins. Food Chem 2010;120:1185-92.

8. Hoffmann J, Nüchter M, Ondruschka B, Wasserscheid P. Ionic liquids and their heating behaviour during microwave irradiation a state of the art report and challenge to assessment. Green Chem 2003;5:296-9.

9. Ayuningtyas IN, Rahmawati M, Sutriyo, Mun'im A. Optimization of ionic liquid-based microwave assisted extraction to obtain transresveratrol from Gnetum gnemon. J Young Pharm 2017;9:446-51.

10. Du F, Xiao X, Li G. Application of ionic liquids in the microwaveassisted extraction of trans-resveratrol from rhizoma polygoni cuspidati. J Chromatogr A 2007;1140:56-62.

11. Bharthare P, Preeti S, Singh P, Archana T. Peanut shell as renewable energy source and their utility in production of ethanol. Int J Adv Res 2014;2:1-12.

12. Li MF, Fan YM, Xu F, Sun RC, Zhang XL. Cold sodium hydroxide/ urea based pretreatment of bamboo for bioethanol production: Characterization of the cellulose rich fraction. Ind Crop Prod 2010;32:551-9

13. García A, Rodríguez-Juan E, Rodríguez-Gutiérrez G, Rios JJ, FernándezBolaños J. Extraction of phenolic compounds from virgin olive oil by deep eutectic solvents (DESs). Food Chem 2016;197:554-61.

14. Cvjetko Bubalo M, Curko N, Tomašević M, Kovačević Ganić K, Radojčić Redovniković I. Green extraction of grape skin phenolics by 
using deep eutectic solvents. Food Chem 2016;200:159-66

15. Jeong KM, Ko JY, Zhao J, Jin Y, Yoo DE, Han SY, et al. Multifunctioning deep eutectic solvents as extraction and storage media for bioactive natural products that are readily applicable to cosmetic products. J Clean Prod 2017;151:87-95.

16. Dai Y, van Spronsen J, Witkamp GJ, Verpoorte R, Choi YH. Natural deep eutectic solvents as new potential media for green technology. Anal Chim Acta 2013;766:61-8.

17. Souto AA, Carneiro MC, Seferin M, Senna MJ, Conz A, Gobbi K. Determination of trans-resveratrol concentrations in Brazilian red wines by HPLC. J Food Compos Anal 2001;14:441-5.

18. Terahadi F, Putri S, Krisanti E, Mulia K. Selected natural deep eutectic solvents for the extraction of $\alpha$-mangostin from mangosteen (Garcinia mangostana L.) pericarp. Int J Technol 2015;6:1211.

19. Liu JL, Li LY, He GH. Optimization of microwave-assisted extraction conditions for five major bioactive compounds from flos sophorae immaturus (cultivars of Sophora japonica L.) using response surface methodology. Molecules 2016;21:296.

20. Chan CH, Yusoff R, Ngoh GC, Kung FW. Microwave-assisted extractions of active ingredients from plants. J Chromatogr A 2011;1218:6213-25

21. Zhang X, Huo F, Liu X, Dong K, He H, Yao X, et al. Influence of microstructure and interaction on viscosity of ionic liquids. Ind Eng Chem Res 2015;54:3505-14.

22. Peng X, Duan MH, Yao XH, Zhang YH, Zhao CJ, Zu YG, et al. Green extraction of five target phenolic acids from Lonicerae japonicae flos with deep eutectic solvent. Sep Purif Technol 2016;157:249-57.

23. Bi W, Tian M, Row KH. Evaluation of alcohol-based deep eutectic solvent in extraction and determination of flavonoids with response surface methodology optimization. J Chromatogr A 2013;1285:22-30.

24. Azwinda NN. A Review on the extraction methods use in medicinal plants, principle, strength and limitation. Med Aromat Plants 2015;4:1-6.

25. Zhang B, Yang R, Liu CZ. Microwave-assisted extraction of chlorogenic acid from flower buds of Lonicera japonica thunb. Sep Purif Technol 2008;62:480-3.

26. Chemat F, Rombaut N, Sicaire AG, Meullemiestre A, FabianoTixier AS, Abert-Vian M. Ultrasound assisted extraction of food and natural products. Mechanisms, techniques, combinations, protocols and applications. A review. Ultrason Sonochem 2017;34:540-60.

27. Mandal V, Mohan Y, Hemalatha S. Microwave assisted extraction-an innovative and promising extraction tool for medicinal plant research. Pharmacog Rev 2007;1:7-18 